\title{
recillunds
}

Revista Científica Mundo de la Investigación y el Conocimiento

Wendy Elizabeth Rivadeneira Manrique a, Viky Allison Rocafuerte Alvarado ${ }^{\text {b; }}$ Carlos Fernando Tello Valdires ${ }^{c}$; Diana Cristina Campoverde Tapia ${ }^{d}$

Tratamiento del síndrome de apnea obstructiva del sueño (SAOS) en pediatría

Treatment of obstructive sleep apnea syndrome (OSAS) in pediatrics

Revista Científica Mundo de la Investigación y el Conocimiento. Vol. 3 núm.3, septiembre, ISSN: 2588-073X, 2019, pp. 314-327

DOI: 10.26820/recimundo/3.(3).septiembre.2019.314-327

URL: http://recimundo.com/index.php/es/article/view/523

Código UNESCO: 3205 Medicina Interna

Tipo de Investigación: Artículo de Revisión

Editorial Saberes del Conocimiento

Recibido: 15/05/2019

Aceptado: 23/06/2019

Publicado: 30/09/2019

Correspondencia: wndyrivadeneira@ hotmail.com

a. Magister en Gerencia de Servicios de Salud; Especialista en Pediatría; Médico; Investigador Independiente; Guayaquil, Ecuador; wndyrivadeneira@ hotmail.com

b. Especialista en Pediatría; Médico; Investigador Independiente; Guayaquil, Ecuador; vikyallison11@ hotmail.com

c. Especialista en Pediatría; Médico; Investigador Independiente; Guayaquil, Ecuador; carlostv33@gmail.com

d. Especialista en Pediatría; Médico; Investigador Independiente; Guayaquil, Ecuador; dianacamp2009@hotmail.com 


\section{Tratamiento del síndrome de apnea obstructiva del sueño (SAOS) en pediatría}

Vol. 3, núm. 3., (2019)

Wendy Elizabeth Rivadeneira Manrique; Viky Allison Rocafuerte Alvarado; Carlos Fernando

Tello Valdires; Diana Cristina Campoverde Tapia

\section{RESUMEN}

El Síndrome de Apnea Obstructiva del Sueño (SAOS) es una alteración importante de la respiración durante el sueño que se caracteriza por la obstrucción parcial, prolongada, de la vía aérea superior y/u obstrucción intermitente completa, la cual interrumpe la ventilación normal durante el sueño y, por ende, sus patrones normales. Entre las complicaciones y consecuencias se encuentran el retraso de crecimiento, hipertensión pulmonar, insuficiencia cardíaca, déficit neurocognitivo, trastornos del aprendizaje y de la conducta, entre otros. El objetivo fundamental de la presente investigación es plasmar las generalidades del tratamiento del SAOS en pediatría. El diseño de investigación que se llevó a cabo es de tipo documental o bibliográfico. Las causas del SAOS son múltiples, entre las estas se destacan las amígdalas agrandadas, el sobrepeso, que el paciente tenga una garganta estrecha o una mandíbula pequeña y factores genéticos. La principal línea de tratamiento está constituida por la adenoamigdalectomía. Se concluye que resulta fundamental que tanto el diagnóstico como la terapéutica del SAOS se encuentre en manos de un equipo multidisciplinario conformado principalmente por pediatras, fonoaudiólogos, otorrinolaringólogos y odontólogos-ortodoncistas y que las re evaluaciones clínicas constituyen un papel fundamental en el éxito del tratamiento en esta etapa infantil.

Palabras Claves: Tratamiento; Síndrome; Apnea; Sueño; Pediatría. 


\title{
Tratamiento del síndrome de apnea obstructiva del sueño (SAOS) en pediatría
}

Vol. 3, núm. 3., (2019)

Wendy Elizabeth Rivadeneira Manrique; Viky Allison Rocafuerte Alvarado; Carlos Fernando Tello Valdires; Diana Cristina Campoverde Tapia

\begin{abstract}
Obstructive Sleep Apnea Syndrome (OSAS) is a major disturbance of breathing during sleep characterized by partial, prolonged obstruction of the upper airway and / or complete intermittent obstruction, which interrupts normal ventilation during sleep. Dream and, therefore, their normal patterns. Complications and consequences include growth retardation, pulmonary hypertension, heart failure, neurocognitive deficit, learning and behavior disorders, among others. The main objective of this research is to capture the generalities of the treatment of OSAS in pediatrics. The research design that was carried out is documentary or bibliographic. The causes of OSA are multiple, among these are the enlarged tonsils, overweight, the patient has a narrow throat or a small jaw and genetic factors. The main line of treatment is constituted by adenoamigdalectomy. It is concluded that it is essential that both the diagnosis and the therapy of OSAS be in the hands of a multidisciplinary team consisting mainly of pediatricians, speech therapists, otolaryngologists and dentists-orthodontists and that clinical reassessments constitute a fundamental role in the success of treatment in This childhood stage.
\end{abstract}

Key Words: Treatment; Syndrome; Apnea; Sleep; Pediatrics. 


\section{Tratamiento del síndrome de apnea obstructiva del sueño (SAOS) en pediatría}

Vol. 3, núm. 3., (2019)

Wendy Elizabeth Rivadeneira Manrique; Viky Allison Rocafuerte Alvarado; Carlos Fernando

Tello Valdires; Diana Cristina Campoverde Tapia

\section{Introducción.}

Los trastornos del sueño generan una gran alerta en la sociedad mundial, dado que la ausencia de sueño o la falta de calidad en el mismo, trae consecuencias desfavorables e importantes para la salud de quienes los padecen. Uno de los principales problemas del sueño está constituido por la apnea del sueño.

La apnea del sueño puede aparecer a cualquier edad, sin embargo, es importante detectarla y tratarla en las primeras etapas de la vida a los fines de evitar sus consecuencias y mejorar la calidad de vida de las personas que la presentan.

Lo que ocurre durante la apnea del sueño en un niño es que la respiración se detiene durante el sueño motivado al estrechamiento de las vías respiratorias o su parcial obstrucción. Existen múltiples causas de la apnea, entre las que se destacan: algunos niños tienen una garganta estrecha. Que por lo general se debe a la presencia de adenoides o amígdalas agrandadas, que bloquean en parte el flujo de aire. Algunos otros factores influyen en la apnea del sueño infantil, tales como una mandíbula pequeña, algunas formas del cielo de la boca (el paladar), o bien, una lengua grande, la cual puede retroceder y bloquear la vía respiratoria, la obesidad puede influir también en la aparición de este trastorno. Por último, un tono muscular deficiente debido a afecciones como síndrome de Down o parálisis cerebral pueden facilitar la aparición de la apnea del sueño infantil. (Biblioteca Nacional de Medicina de los Estados Unidos, 2017) 


\section{Tratamiento del síndrome de apnea obstructiva del sueño (SAOS) en pediatría}

Vol. 3, núm. 3., (2019)

Wendy Elizabeth Rivadeneira Manrique; Viky Allison Rocafuerte Alvarado; Carlos Fernando Tello Valdires; Diana Cristina Campoverde Tapia

"El síndrome de apnea del sueño es un problema común que afecta a cerca de un 2\% de todos los niños, incluyendo muchos que no han sido diagnosticados". Es importante el diagnóstico y tratamiento lo más temprano posible, ya que esta afección conduce a diversos problemas en la salud del niño. (Academia Americana de Pediatras, 2015)

Por su parte, Alonso \& Mínguez (2018) describen al síndrome de apnea obstructiva del sueño (SAOS) en niños como "una alteración de la respiración durante el sueño, caracterizada por una obstrucción parcial, prolongada, de la vía aérea superior y/u obstrucción intermitente completa, que interrumpe la ventilación normal durante el sueño y los patrones normales del sueño”. (p. 422)

Asimismo, es importante destacar que existen diferencias entre la apnea obstructiva del sueño en niños y la apnea del sueño en adultos y que radica en que los adultos tienen somnolencia durante el día por lo general, mientras que los niños pueden llegar hasta a presentar problemas de conducta. En los adultos la causa subyacente suele ser la obesidad. Por otra parte, la causa más común en niños es el agrandamiento de las adenoides y las amígdalas. (Mayo Clinic, 2019)

La importancia de tener un sueño de calidad se centra en que a pesar de que el individuo está inconsciente mientras duerme, las funciones de su cerebro y cuerpo se mantienen activas, realizando múltiples funciones vitales para la salud. En consecuencia, cuando no se tiene un sueño de calidad, además de sentirse cansado, podría afectar la salud mental y física de la persona. (Biblioteca Nacional de Medicina de los Estados Unidos, 2019) 


\section{Tratamiento del síndrome de apnea obstructiva del sueño (SAOS) en pediatría}

Vol. 3, núm. 3., (2019)

Wendy Elizabeth Rivadeneira Manrique; Viky Allison Rocafuerte Alvarado; Carlos Fernando

Tello Valdires; Diana Cristina Campoverde Tapia

Para Cuestas \& Boccio (2015) “la prevalencia del SAOS en la población infantil es del 12\%, y es más frecuente entre los 2 y 8 años". Entre sus principales complicaciones y consecuencias se encuentran: retraso de crecimiento, hipertensión pulmonar, hipertensión arterial sistémica, insuficiencia cardíaca, déficit neurocognitivo, trastornos del aprendizaje y de la conducta. (p. 32,33).

El objetivo del estudio consiste en revisar y plasmar el manejo del síndrome de apnea obstructiva del sueño en pediatría, desde la perspectiva de las causas y las líneas actuales de tratamientos: quirúrgicos, presión positiva continua sobre la vía aérea, farmacológico, oxigenoterapia, tratamiento odontológico-ortopédico y tratamiento conservador.

\section{Materiales y Métodos.}

Para desarrollar el estudio se usaron computadores personales con conexión a internet, con el propósito de recopilar información, revisar y plasmar los tratamientos del síndrome de apnea obstructiva del sueño en pediatría, en razón de lo cual, la investigación se enmarca en un diseño documental o bibliográfico.

Tal investigación está limitada a la búsqueda y revisión sistemática de material bibliográfico, al cual se accedió por medio del uso de bases de datos, entre las que figuraron: PubMed, MedlinePlus, Mayo Clinic, Biblioteca Virtual de la Salud (BVS), SciELO, Dialnet y ELSEVIER, entre otras; las cuales permitieron elaborar una síntesis de la evidencia disponible.

La búsqueda se llevó a cabo de forma aleatoria y consecutiva, mediante las expresiones "síndrome de apnea del sueño obstructiva en niños", "apnea del sueño"; "apnea del sueño en 


\section{Tratamiento del síndrome de apnea obstructiva del sueño (SAOS) en pediatría}

Vol. 3, núm. 3., (2019)

Wendy Elizabeth Rivadeneira Manrique; Viky Allison Rocafuerte Alvarado; Carlos Fernando Tello Valdires; Diana Cristina Campoverde Tapia

pediatría" y "tratamiento de apnea del sueño en la infancia". Dicha búsqueda resultó en cientos de registros bibliográficos, los cuales se filtraron tomando en cuenta criterios de idioma español, correlación temática, relevancia y fecha de publicación en los últimos once años.

De la correspondiente lectura crítica y análisis de la evidencia científica recopilada, resultó en el contenido de la presente investigación.

\section{Resultados.}

Causas del Síndrome de Apnea Obstructiva del Sueño (SAOS) en Pediatría

Existen factores de riesgo que son elementos que elevan la probabilidad de que el niño presente el SAOS, este puede presentar uno o una concurrencia de varios factores.

“Son diversas las causas por las que un niño puede sufrir apnea del sueño. Las amígdalas agrandadas o las vegetaciones provocan, en ocasiones, alteraciones en la respiración mientras el pequeño está durmiendo". Otros factores importantes que predisponen la aparición del SAOS es el sobrepeso, que el paciente tenga una garganta estrecha o una mandíbula pequeña, así como alguna malformación en el paladar, la lengua o las vías respiratorias. (Sociedad Española de Otorrinolaringología y Cirugía de Cabeza y Cuello, s.f.)

Para la American Thoracic Society (2009) otros factores de riesgo lo constituyen problemas con el tono muscular, que son casos donde los músculos de la garganta se relajan y son capaces de bloquear la vía aérea, casos más frecuentes en afecciones tales como la distrofia muscular y parálisis cerebral. Asimismo, el SAOS puede derivar de síndromes genéticos, tales como el síndrome de Down y el síndrome de Prader-Willi. Por otro lado, los problemas 


\section{Tratamiento del síndrome de apnea obstructiva del sueño (SAOS) en pediatría}

Vol. 3, núm. 3., (2019)

Wendy Elizabeth Rivadeneira Manrique; Viky Allison Rocafuerte Alvarado; Carlos Fernando

Tello Valdires; Diana Cristina Campoverde Tapia

cerebrales para controlar la respiración pueden afectarla durante el sueño. Por último, los antecedentes familiares también juegan un papel importante en la predisposición de este trastorno, ya que puede ser genética, en consecuencia, la probabilidad de que un niño padezca SAOS aumenta con la presencia del problema en un familiar. (p. 5)

\section{Tratamientos para el SAOS en Pediatría}

\section{Tratamiento quirúrgico}

\section{Adenoamigdalectomía}

Se trata del tratamiento de primera línea del SAOS y es un procedimiento quirúrgico que consiste en la remoción tanto de las amigdalas como de las adenoides, con la finalidad de abrir las vías aereas superiores del paciente y proporcionar una mejor función de la respiración.

Villa, et. al (2008) mencionan en su trabajo que la hipertrofia adenoamigdalar es fundamentalmente la causa del SAOS en la mayoría de los niños, por tanto, su tratamiento principal es la adenoamigdalectomía. En algunos casos es posible que exista contraindicación para llevar a cabo esta cirugía o que no se haya producido mejoría completa tras la misma, en estos casos deben aplicarse otro tipo de tratamientos. "La adenoamigdalectomía ha demostrado resolver los trastornos respiratorios y los síntomas nocturnos en el 75 a 100\% de los niños sin otra patología de base y se considera el tratamiento de primera elección en niños con rasgos craneofaciales normales". (p. 122) 


\section{Tratamiento del síndrome de apnea obstructiva del sueño (SAOS) en pediatría}

Vol. 3, núm. 3., (2019)

Wendy Elizabeth Rivadeneira Manrique; Viky Allison Rocafuerte Alvarado; Carlos Fernando Tello Valdires; Diana Cristina Campoverde Tapia

\section{Uvulofaringopalatoplastia}

Este procedimiento quirúrgico se ha empleado en niños con SAOS y retraso psicomotor los cuales presentaban paladar y úvula hipertróficos. Su utilidad se remite a aquellos casos en que la obstrucción principal se localiza en el paladar blando. Uno de sus 'principales riesgos es la insuficiencia velofaríngea, la cual se presenta con mayor frecuencia en niños. (Villa, et. al, 2008, p. 123)

\section{Cirugía maxilofacial}

Se encuentra indicada en casos específicos, tales como malformación del macizo facial o de la base del cráneo, asimismo en los síndromes de craneosinostosis (Crouzon, Apert, Pfieffer), en el síndrome de Pierre-Robin y síndrome de Down, en retrognatia, atresia de coanas o alteraciones del tabique nasal. (Villa, et. al, 2008, p. 123)

\section{Presión Positiva Continua sobre la vía Aérea (CPAP)}

Este tratamiento consiste en el uso de pequeñas máquinas que soplan aire por medio de un tubo y máscara debidamente colocada, bien sea sobre la nariz, o la nariz y la boca del niño. Este aire es enviando hacia la parte posterior de la garganta con la finalidad de abrir y mantener abiertas las vías aéreas y proporcionar una mejor respiración.

Este tratamiento en pediatría, constituye la segunda línea de tratamiento del SAOS, y se encuentra indicado en aquellos casos de SAOS residual, es decir, provenientes de adenoamigdalectomía, en SAOS relacionado con obesidad del niño, en casos de alteraciones 


\section{Tratamiento del síndrome de apnea obstructiva del sueño (SAOS) en pediatría}

Vol. 3, núm. 3., (2019)

Wendy Elizabeth Rivadeneira Manrique; Viky Allison Rocafuerte Alvarado; Carlos Fernando

Tello Valdires; Diana Cristina Campoverde Tapia

craneofaciales o enfermedades neuromusculares que se encuentran añadidas a la hipertrofia adenoamigdalar o sin hipertrofia adenoamigdalar. Para este tratamiento resulta fundamental realizar controles periódicos, dado que los niños se encuentran en crecimiento, esto exige nuevos y cambiantes requerimientos que se ajusten o vayan a la par con su crecimiento y desarrollo, como la presión y el ajuste de la mascarilla. Asimismo, es importante la detección precoz de efectos secundarios que se deriven del tratamiento. Por último, cabe destacar que la CPAP no es un tratamiento curativo, en virtud de lo cual, un adecuado cumplimiento resulta imprescindible. (Alonso \& Mínguez, 2018, p. 429)

\section{Oxigenoterapia}

La oxigenoterapia es un tratamiento capaz de proporcionar oxígeno adicional al paciente a los fines de que pueda cumplir con todas sus funciones vitales. Es recomendable en casos crónicos de SAOS cuando este evita que le llegue el oxígeno necesario para el adecuado funcionamiento del organismo.

Para Luna et. al (2009) constituye una herramienta básica para el tratamiento de la insuficiencia respiratoria, tanto aguda como crónica. Tiene como objetivo fundamental "tratar o prevenir la hipoxemia, tratar la hipertensión pulmonar y reducir el trabajo respiratorio y miocárdico. En situaciones agudas, su utilidad está ampliamente aceptada y en situaciones crónicas se ha extendido de forma importante". (p. 162)

\section{Tratamiento conservador}




\section{Tratamiento del síndrome de apnea obstructiva del sueño (SAOS) en pediatría}

Vol. 3, núm. 3., (2019)

Wendy Elizabeth Rivadeneira Manrique; Viky Allison Rocafuerte Alvarado; Carlos Fernando Tello Valdires; Diana Cristina Campoverde Tapia

Es recomendable la pérdida de peso, en los casos de niños con SAOS que tienen sobrepeso u obesidad, no obstante, dado que este proceso no resulta inmediato es importante alternar con otro tratamiento hasta que el paciente llegue a su peso ideal.

Schwab et al. citado por Guilleminault \& Parejo (2017) en su trabajo, "mostraron que la obesidad, como efecto secundario, agranda la lengua, lo que lleva a la apnea obstructiva del sueño". (p. 15)

\section{Tratamiento farmacológico}

“Los esteroides nasales tópicos, como la fluticasona (Dymista, Flonase Allergy Relief, Xhance) y la budesonida (Rhinocort), pueden aliviar los síntomas de la apnea del sueño en algunos niños con síndrome de apnea obstructiva del sueño leve”. En aquellos casos de niños con alergias, se recomienda el uso de montelukast (Singulair) a los fines de aliviar los síntomas, puede ser administrado solo o acompañado con esteroides nasales. (Mayo Clinic, 2019)

\section{Tratamiento odontológico-ortopédico}

Para Kocuta (2017) la finalidad del tratamiento ortopédico en pacientes que presentan SAOS es armonizar las estructuras con la finalidad de facilitar la reeducación de la respiración. "Los tratamientos habituales son aquellos que actúan a nivel de la sutura medio palatina. Esto produce un aumento transversal del maxilar superior $\mathrm{y}$, los que generan, un adelantamiento mandibular". Estos tratamientos sobre la sutura medio palatina varían dependiendo del estadio de osificación de la misma, donde en edades tempranas es recomendable realizar ensanche maxilar con placas activas que actúan sobre la sutura palatina. Este tratamiento cierra con el uso de 


\section{Tratamiento del síndrome de apnea obstructiva del sueño (SAOS) en pediatría}

Vol. 3, núm. 3., (2019)

Wendy Elizabeth Rivadeneira Manrique; Viky Allison Rocafuerte Alvarado; Carlos Fernando

Tello Valdires; Diana Cristina Campoverde Tapia

aparatología funcional de avance mandibular con el objeto de conseguir mejoría en el flujo de aire en la faringe, en donde se adelanta la lengua acompañando a la mandíbula, con elevación del hioides y, por ende, dilatación de los músculos faríngeos. Dentro de los aparatos usados para este tratamiento se encuentran: activadores, Bionator, activador de Klammt. (p. 24, 25)

\section{Conclusión.}

En principio es importante puntualizar que un buen tratamiento es fundamental para evitar complicaciones futuras y brindar en una edad temprana al paciente una mejor calidad de vida, en razón de lo cual, es importante que tanto el diagnóstico como la terapéutica del SAOS se encuentre en manos de un equipo multidisciplinario conformado principalmente por pediatras, fonoaudiólogos, otorrinolaringólogos y odontólogos-ortodoncistas, quienes serán os encargados de manera conjunta de puntualizar las causas que originan el problema y brindar el tratamiento oportuno y eficaz al problema.

El tratamiento del SAOS en pediatría, debe ser específico y único para cada paciente y en muchos casos, debe ser combinado, tratando de obtener los mejores y más rápidos resultados positivos en el paciente por medio de la resolución de estos problemas de respiración durante la fase de sueño.

El tratamiento varía según la causa que origina el trastorno en el niño, siendo la hipertrofia adenoamigdalina la causa más común de SAOS, la adenoamigdalectomía se constituye como el tratamiento de primera línea. No obstante, puede que por sí sola no resuelva el problema, en cuyo caso es fundamental la aplicación de un tratamiento adicional. 


\section{Tratamiento del síndrome de apnea obstructiva del sueño (SAOS) en pediatría}

Vol. 3, núm. 3., (2019)

Wendy Elizabeth Rivadeneira Manrique; Viky Allison Rocafuerte Alvarado; Carlos Fernando Tello Valdires; Diana Cristina Campoverde Tapia

Por último, es imperioso, sobre todo por ser esta etapa infantil en donde surgen acelerados y constantes variaciones por el crecimiento y desarrollo, la reevaluación clínicamente de este paciente, preferiblemente a los seis a doce meses después de haber iniciado el tratamiento, a los fines de verificar la efectividad de su terapéutica y realizar, de ser necesario, reajustes en el mismo, con el propósito de garantizar que no surjan alteraciones en la salud del niño a mediano o largo plazo.

\section{Bibliografía.}

Academia Americana de Pediatras. (21 de Noviembre de 2015). Healthy Children. Recuperado el 12 de Septiembre de 2019, de https://www.healthychildren.org/Spanish/agesstages/baby/sleep/Paginas/Sleep-Apnea-Detection.aspx

Alonso, M., \& Mínguez, R. (2018). Trastornos respiratorios del sueño. Síndrome de apneahipoapnea del sueño en la infancia. Pediatría Integral, 22(8), 422-436. Recuperado el 13 de Septiembre de 2019, de https://www.pediatriaintegral.es/wpcontent/uploads/2018/xxii08/07/n8-422-436_RamonaMinguez.pdf

American Thoracic Society. (2009). Síndrome de apnea obstructiva del sueño en los niños. Revista norteamericana de medicina respiratoria y cuidados intensivos, 180, 5-6. Recuperado el 15 de Septiembre de 2019, de https://www.thoracic.org/patients/patientresources/resources/spanish/obstructive-sleep-apnea-in-children.pdf

Biblioteca Nacional de Medicina de los Estados Unidos. (09 de Mayo de 2017). MedlinePlus. $\begin{array}{llllll}\text { Recuperado el } 12 \text { de } & \text { Septiembre }\end{array}$ https://medlineplus.gov/spanish/ency/article/007660.htm

Biblioteca Nacional de Medicina de los Estados Unidos. (06 de Junio de 2019). MedlinePlus. $\begin{array}{llllll}\text { Recuperado el } & 13 \text { de } & \text { Septiembre } & \text { de }\end{array}$ https://medlineplus.gov/spanish/sleepdisorders.html

Cuestas, G., \& Boccio, C. (2015). Factores morfogenéticos del síndrome de apneas obstructivas del sueño en niños. Revista FASO, 22(2), 32-35. Recuperado el 14 de Septiembre de 2019, de http://faso.org.ar/revistas/2015/2/6.pdf 


\section{Tratamiento del síndrome de apnea obstructiva del sueño (SAOS) en pediatría}

Vol. 3, núm. 3., (2019)

Wendy Elizabeth Rivadeneira Manrique; Viky Allison Rocafuerte Alvarado; Carlos Fernando

Tello Valdires; Diana Cristina Campoverde Tapia

Guilleminault, C., \& Parejo, K. (2017). Historia del síndrome de apnea-hipopnea obstructiva del sueño (SAHOS). Rev. Fac. Med., 65, 11-16. Recuperado el 14 de Septiembre de 2019, de http://www.scielo.org.co/pdf/rfmun/v65s1/0120-0011-rfmun-65-s1-00011.pdf

Kocuta, M. (2017). Apnea en niños: diagnóstico y tratamiento. RAAO, 52(2), 21-27. Recuperado el 13 de Septiembre de 2019, de https://www.ateneoodontologia.org.ar/articulos/lvii02/articulo2.pdf

Luna, M., De la Cruz, O., Cortell, I., Martínez, M., Barrio, M., Pérez, E., \& Pérez, J. (2009). Fundamentos de la oxigenoterapia en situaciones agudas y crónicas: indicaciones, métodos, controles y seguimiento. Anales de Pediatría, 71(2), 161-174. Recuperado el 14 de Septiembre de 2019, de https://www.analesdepediatria.org/es-pdfS1695403309003294

Mayo Clinic. (15 de Enero de 2019). Mayo Clinic. Recuperado el 14 de Septiembre de 2019, de https://www.mayoclinic.org/es-es/diseases-conditions/pediatric-sleep-apnea/symptomscauses/syc-20376196

Sociedad Española de Otorrinolaringología y Cirugía de Cabeza y Cuello. (s.f.). SEORL CCC. Recuperado el 14 de Septiembre de 2019, de https://seorl.net/apnea-sueno-infantil/

Villa, J., Martínez, C., Pérez, G., Cortell, I., Gómez, D., Alvarez, D., \& González, Y. (2008). Síndrome de apneas-hipopneas del sueño. España: Asociación Española de Pediatría. Recuperado el 13 de Septiembre de 2019, de https://www.aeped.es/sites/default/files/documentos/7_4.pdf 